\title{
Telomerase antagonist imetelstat increases radiation sensitivity in esophageal squamous cell carcinoma
}

\author{
Xuping Wu ${ }^{1, *}$, Jing Zhang ${ }^{2, *}$, Sijun Yang ${ }^{3}$, Zhihui Kuang ${ }^{2}$, Guolei Tan ${ }^{1}$, Gang Yang ${ }^{1}$, \\ Qichun Wei ${ }^{4}$, Zhigang Guo ${ }^{2}$ \\ ${ }^{1}$ The Second Hospital of Nanjing Affiliated to Medical School of Southeast University, Nanjing 210003, China \\ ${ }^{2}$ Jiangsu Key Laboratory for Molecular and Medical Biotechnology, College of Life Sciences, Nanjing Normal University, \\ Nanjing 210023, China \\ ${ }^{3}$ ABSL-3 Laboratory at The Center for Animal Experiment and State Key Lab of Virology, Wuhan University, Wuhan 430071, \\ China \\ ${ }^{4}$ Department of Radiation Oncology, Second Affiliated Hospital, Zhejiang University School of Medicine, Hangzhou 31009, \\ China \\ *These authors contributed equally to this work and as the first author
}

Correspondence to: Zhigang Guo, email: guozgang@gmail.com

Xuping Wu, email: xuping_wu@yahoo.com

Keywords: esophageal squamous cell carcinoma, telomerase, radiosensitization, apoptosis

Received: January 17, 2016

Accepted: January 03, 2017

Published: January 13, 2017

\section{ABSTRACT}

The morbidity and mortality of esophageal cancer is one of the highest around the world and the principal therapeutic method is radiation. Thus, searching for sensitizers with lower toxicity and higher efficiency to improve the efficacy of radiation therapy is critical essential. Our research group has previously reported that imetelstat, the thio-phosphoramidate oligonucleotide inhibitor of telomerase, can decrease cell proliferation and colony formation ability as well as increase DNA breaks induced by radiation in esophageal cancer cells. Further study in this project showed that imetelstat significantly sensitized esophageal cancer cells to radiation in vitro. Later study showed that imetelstat leads to increased cell apoptosis. We also measured the expression level of several DNA repair and apoptosis signaling proteins. pS345 CHK1, Y-H2AX, p53 and caspase 3 expression were up-regulated in imetelstat treated cells, identifying these factors as molecular markers. Mouse in vivo model using imetelstat at clinically achievable concentrations and fractionated irradiation scheme yielded results demonstrating radiosensitization effect. Finally, TUNEL assay, caspase 3 and Ki67 staining in tumor tissue proved that imetelstat sensitized esophageal cancer to radiation in vivo through promoting cell apoptosis and inhibiting cell proliferation. Our study supported imetelstat increase radiation sensitivity of esophageal squamous cell carcinoma through inducing cell apoptosis and the specific inhibitor of telomerase might serve as a potential novel therapeutic tool for esophageal squamous cell carcinoma therapy.

\section{INTRODUCTION}

Esophageal carcinoma is a common malignant tumor of digestive system with the mortality rate ranking the sixth in the world [1]. Surgical resection is preferred for patients with early esophageal cancer. However, patients in clinical mainly suffer from advanced esophageal cancer [2-3] which is often accompanied by lymph node metastasis or other complications. Radiation is one of most important and effective therapeutic methods for esophageal cancer. However, esophageal cancer belongs to the moderately sensitive tumors to radiotherapy. The 3-year overall survival rate has been reported to be only about $20 \%$ after radiotherapy alone while the recurrence rate is as high as $60 \%-80 \%$ [4]. Although increasing the irradiation intensity could improve the therapeutic effect, the normal tissues might be also damaged and radioresistance might be another major challenge resulting in locoregional 
recurrence and metastasis [5]. Thus, it is essential to develop radiation sensitizer with high efficiency and low toxicity for the treatment of esophageal cancer [6].

It has been extensively reported that targeting telomerase is effective for cancer treatment [7] and the most direct way is to inhibit the activity of telomerase is targeting telomerase RNA (hTR) or reverse transcriptase protein subunit (hTERT). Imetelstat, a 13-mer oligonucleotide which is covalently modified with lipid extensions, competitively suppresses enzymatic activity of telomerase [8]. It has been found to target the template region of hTR and serve as an oligonucletide template antagonist [9]. Covalently bonded liposomes are prone to be uptaken by cell, which ensures a better and reliable bioavailability. Meanwhile, thiophosphoramidate internucleoside linkages in imetelstat provide a longer half-life for the inhibitor in vivo [10-11]. Furthermore, it has been reported that telomerase activity was remarkably elevated and inhibition of telomerase blocks proliferation of esophageal adenocarcinoma cells both in vitro and in vivo [12], while there was no report focusing on esophageal squamous cell carcinoma.

Radiation produce large amounts of free radicals which further leads to DNA breakage. DNA double strand breaks (DSBs) is not only the most serious injury caused by radiation, but also the basis of radiation to kill tumor cells [13]. Once DSBs is induced, cells themselves react quickly and activate DNA damage responses which recruit large amounts of protein such as ATM, $\gamma-\mathrm{H} 2 \mathrm{AX}, \mathrm{p} 53$ to sense, amplify and transduce DNA damage signal rapidly [14]. Eventually, cells respond to these signals to protect themselves, including cell cycle checkpoints, regulation of gene expression and cell apoptosis.

In the present study, we investigated the increased radiosensitization effect of imetelstat on esophageal squamous cell carcinoma in vitro and in vivo. The effect of imetelstat on proliferation and radiosensitization was correlated with its ability to increase cell apoptosis.

\section{RESULTS}

\section{Imetelstat enhanced Kyse410 and Kyse520 sensitivity to radiotherapy}

Prior investigations have revealed that inhibitor imetelstat treated esophageal squamous cells is able to increase the number of double-strand breaks induced by high energy X-Ray [9]. To further study the radiosensitization of imetelstat on esophageal squamous carcinoma cells, the present study was carried out on the basis of previous researches. As shown in Figure 1A, exposure to imetelstat alone resulted in $69.7 \%$ cell survival for Kyse410 and 75.9\% cell survival for Kyse520 compare to sense treatment. Kyse410 and Kyse520 cells were exposed to radiation of $0,2,4,6$ and 8 Gy X-ray in the presence or absence of imetelstat to study cell survival. Cell survival after 2, 4, 6 and 8 Gy irradiation of Kyse410 was $64.7 \%, 39.1 \%, 3.9 \%$ and $3.3 \%$, whereas the expected additive survival should be $45.0 \%, 27.2 \%, 2.7 \%$ and $2.3 \%$. However, the achieved cell survival after combination was less, 30.1\%, 20.3\%, 3.3\% and 0.9\%. For Kyse 520 , the expected additive cell survival is approximately $61.4 \%, 38.3 \%, 17.4 \%$ and $6.5 \%$ theoretically. Whereas, the achieved cell survival of Kyse520 exposed to 2, 4, 6 and 8 Gy irradiation and imetelstat combination was $47.6 \%, 30.2 \%, 11.0 \%$ and $5.5 \%$. Figure $1 \mathrm{~B}$ showed the survival curves of Kyse410 and Kyse520 after exposure to X-ray combined with the treatment of $5 \mu \mathrm{M}$ imetelstat, which reflected the radiosensitizing effect. As indicated, imetelstat treated cells were normalized to sense treated controls, the figure only reflected the raidosensitizing effect. We observed that imetelstat could radiosensitize both Kyse410 and Kyse520 cells, the obtained SERs were 1.9 and 1.6 , respectively.

\section{Imetelstat increases cell apoptosis of Kyse410 and Kyse520 cells}

Apoptosis, a mode of cell death in response to radiation, is an important method for cancer therapy. To test whether Kyse410 and Kyse520 cell apoptosis was enhanced by imetelstat, annexin V - FITC staining were applied. Temozolomide (TMZ) can form DNA double strand breaks and induce cell apoptosis. In later studies, TMZ was selected to mimic the function of radiation in vitro. As shown in Figure 2, TMZ significantly increased apoptosis percentage for both cell lines compared with the control group $(p<0.05)$. Apoptosis rate for Kyse 410 and Kyse 520 cells was approximately $24 \%$ and $14 \%$ with the exposure to $\mathrm{TMZ} / \mathrm{sense}$. Imetelstat administration increased the apoptosis percentage to $38 \%$ for Kyse 410 and $18 \%$ for Kyse 520 , significantly higher than those treated with sense $(p<0.05)$.

Cells react to DSBs by triggering the DNA damage checkpoint response, which arrests cell-cycle progression until the DNA damage has been removed. CHK1 is a multifunctional protein kinase that plays essential roles in cell survival and cell cycle checkpoints. As shown in Figure 3A, Kyse cells were treated with $10 \mu \mathrm{M}$ TMZ and showed increased phosphorylation of CHK1. Furthermore, imetelstat treatment could increase CHK1 phosphorylation compare with sense treatment. The magnitude and rate of DNA double-strand breaks repair were assessed by the analysis of $\gamma-\mathrm{H} 2 \mathrm{AX}$ using western blot. Cell treated with TMZ over a dose range of 5, 10 and $20 \mu \mathrm{M}$ showed increased $\gamma$-H2AX in the imetelstat-treated samples for both Kyse410 and Kyse520 cells (Figure 3B and 3C), while the relative protein levels in Kyse410 cell was higher than that in Kyse520 cells. Human tumor suppressor gene p53 is known to be implicated in DNA repair and induce cell apoptosis. As illustrated in Figure 3B, the expression of p53 in Kyse410 cells was up-regulated with the treatment of imetelstat. Besides, caspase family 

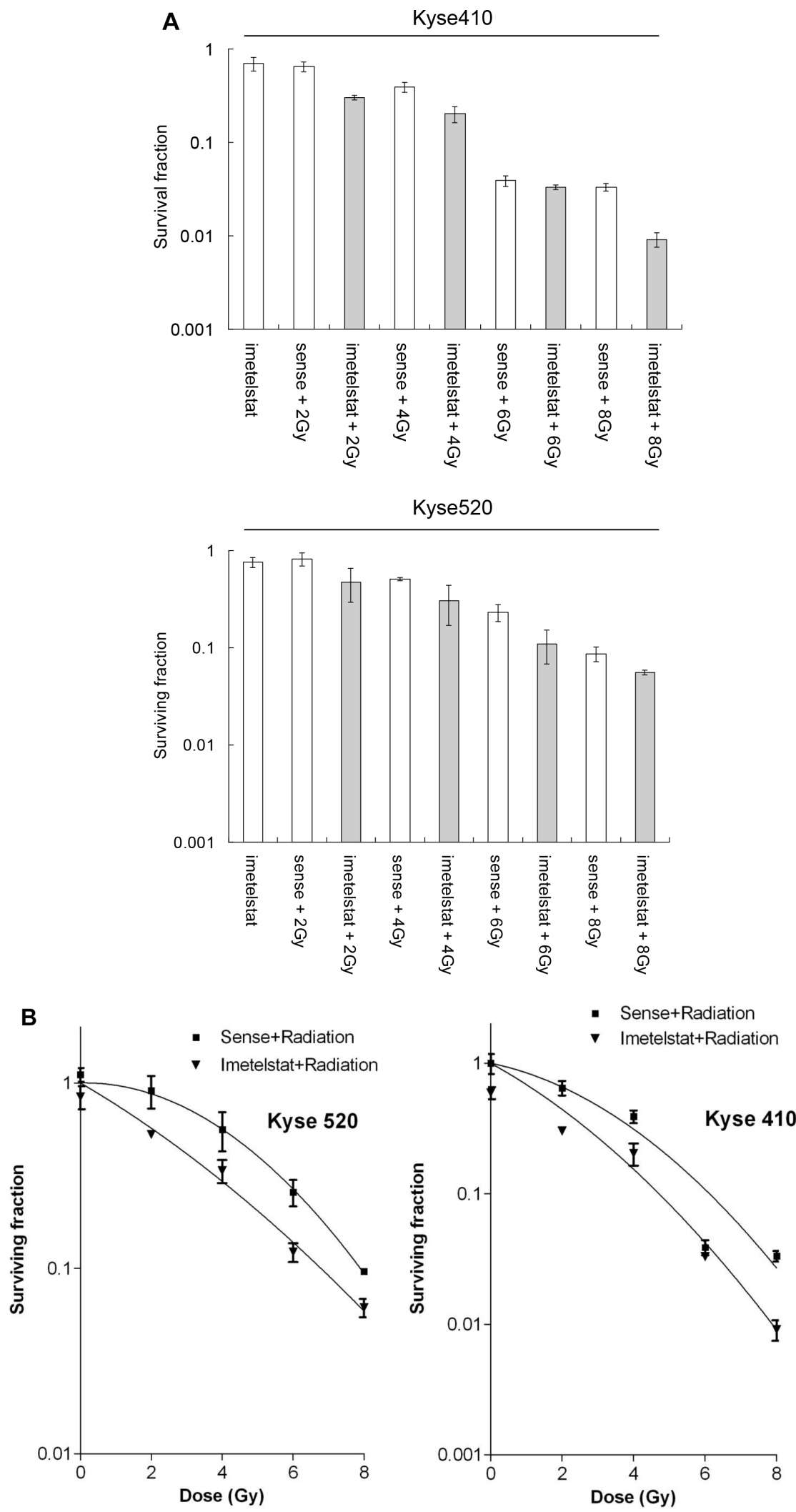

Figure 1: Imetelstat radiosensitizes Kyse410 and Kyse520 cells by using clonogenic cell survival following imetelstat and/or X-ray irradiation (2, 4, 6 and 8 Gy). (A) shows survival fraction as a function of imetelstat, radiation dose and their combination. (B) shows survival plots as a function of radiation dose with or without imetelstat. Survival data were fitted to $S=\exp \left(-\alpha \mathrm{D}-\beta \mathrm{D}^{2}\right)$ where $\mathrm{D}$ is the dose in Gy and $\alpha$ and $\beta$ are fitting parameters, $n \geq 6$, Error bars represent standard deviation. All imetelstat treatment had $P$-values less than 0.05 except imetelstat +6 Gy treatment for Kyse 410 . 
plays a critical important role in mediating the process of apoptosis, wherein caspase 3 is key execution molecule. It was also found that the expression of caspase 3 in Kyse 410 and Kyse520 cells were up-regulated with the treatment of $5 \mu \mathrm{M}$ imetelstat (Figure 3B and 3C).

\section{Imetelstat sensitizes esophageal cancer cells to radiation in vivo}

To study whether telomerase inhibition and telomere dysfunction was associated with a progressive impairment in the cell growth in vivo as well as in vitro, the tumor growth curve was pictured. Kyse cells were pretreated with imetelstat and tumors in nude mice were subjected to 2 Gy of irradiation for 5 consecutive days. As seen in Figure 4, the volume of tumor in nude mice treated with $\mathrm{X}$-ray exhibited a decrease in contrast with the untreated and sense group. As a telomerase inhibitor, imetelstat itself could significantly inhibit the growth of tumor compared with those in the sense group. More importantly, Kyse520 in imetelstat/radiation group showed a lag in tumor growth compared with mice receiving 2 Gy of irradiation alone or irradiation with sense, which confirmed that imetelstat made tumor more sensitive to radiotherapy.

\section{Imetelstat increases cell apoptosis and decreases cell proliferation in vivo}

Pathological changes of esophageal squamous carcinoma were evaluated by $H \& E$ staining. As seen in Figure 5, tumor samples from the untreated group were tightly aligned with a large blue-hued nucleus. Besides, samples from mice subjected to imetelstat alone displayed more vacuoles in contrast with those in the sense group. Tumor isolated from mice in the imetelstat/radiation group showed condensed cytoplasm and desmosome complexes, fragmented nuclear, separated cells in comparison with those in the sense/radiation group, suggesting the potential sensitizing effect.

Apoptotic cells were labeled by TUNEL assay as shown in Figure 5. Although TUNEL staining for tumor treated with sense or imetelstat showed relatively small number of apoptotic cells, the enhanced staining intensity in imetelstat group was still stronger than that in the sense group. Moreover, tumor exposed to the combination of radiation and sense also showed less apoptosis staining than those subjected to imetelstat/radiation. The apoptosis induced by imetelsat and radiation was further analyzed by using caspase 3 immunofluorescence staining. The expression of caspase 3 in radiation-treated tumor in vivo was up-regulated, while imetelstat promoted radiationinduced cell apoptosis as reflected by the further upregulated level of caspase3 (Figure 5). More importantly, the expression of caspase 3 in imetelstat/radiation group was much higher than that in the sense/radiation group, which implies more apoptosis. Tumor subjected to sense or imetelstat only showed almost no expression of caspase 3, which confirmed that imetelstat was not toxic to mice in vivo and the principal function of it was enhancing tumor sensitivity to radiation therapy.

The expression of $\mathrm{Ki} 67$ is indispensable in cell proliferation. Radiation-treated mice showed the down-
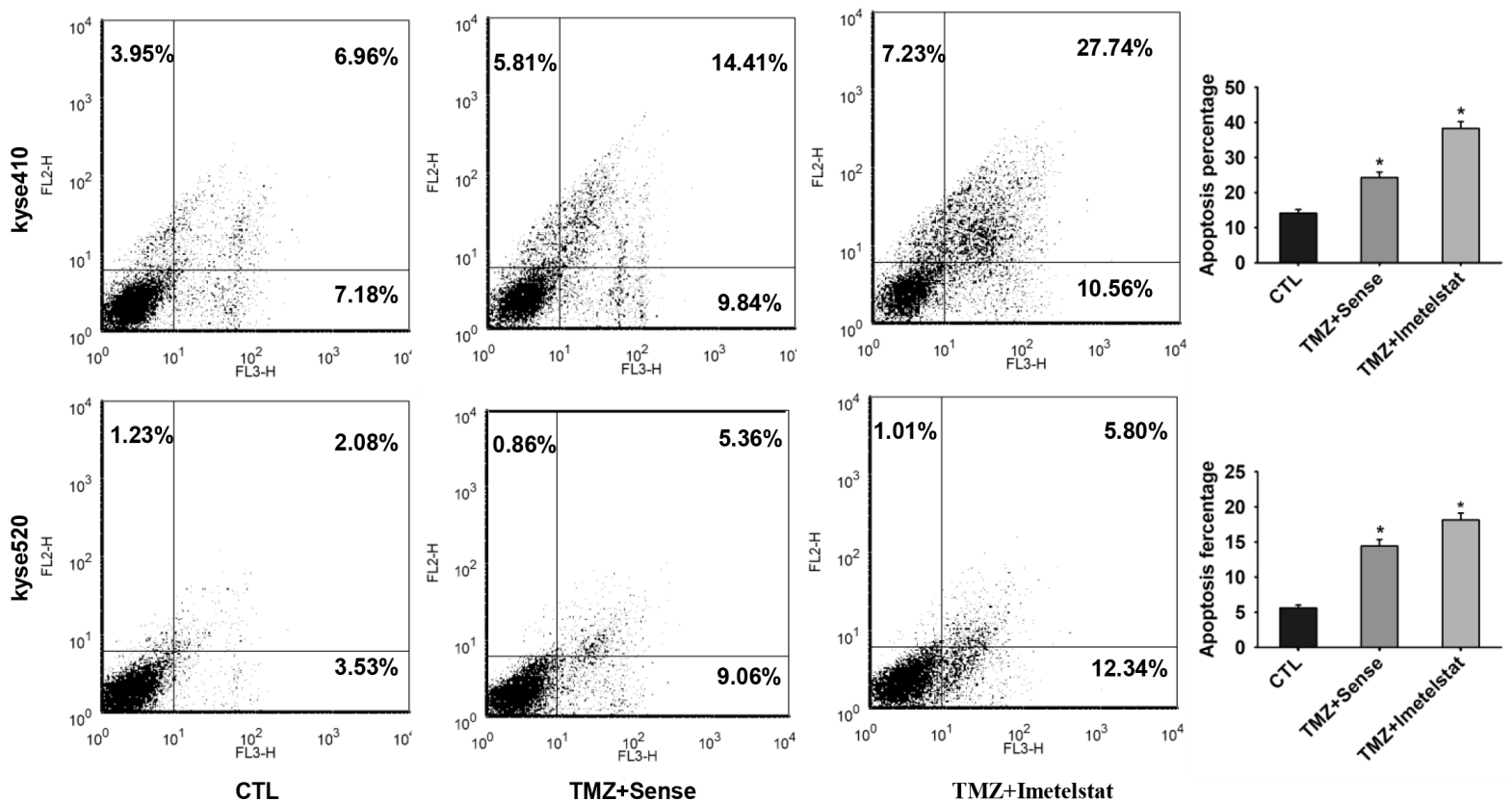

Figure 2: Imetelstat increases cell apoptosis of Kyse410 and Kyse520 cell. Kyse410 and Kyse520 cells were incubated and treated with $5 \mu \mathrm{M}$ sense or imetelstat for $72 \mathrm{~h}$ and at the same time exposed to $10 \mu \mathrm{M}$ TMZ. Results of early and late apoptosis were added together to calculate the total amount of apoptosis. Values represent the mean $\pm \mathrm{SD}$ and are representative of three independent experiments. ${ }^{*} p<0.05$, statistical significance was established by $t$ test. 
A

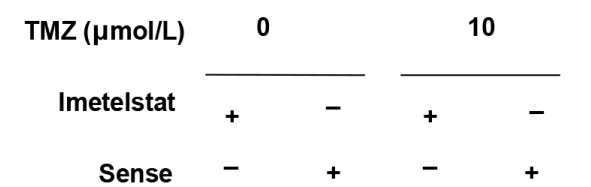

pS345 CHK1
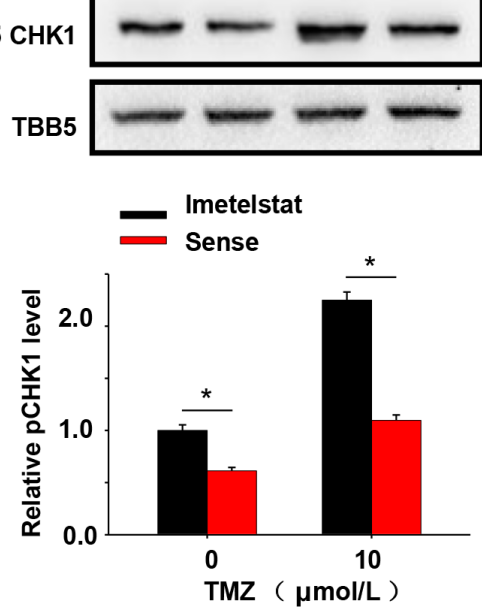

kyse 520
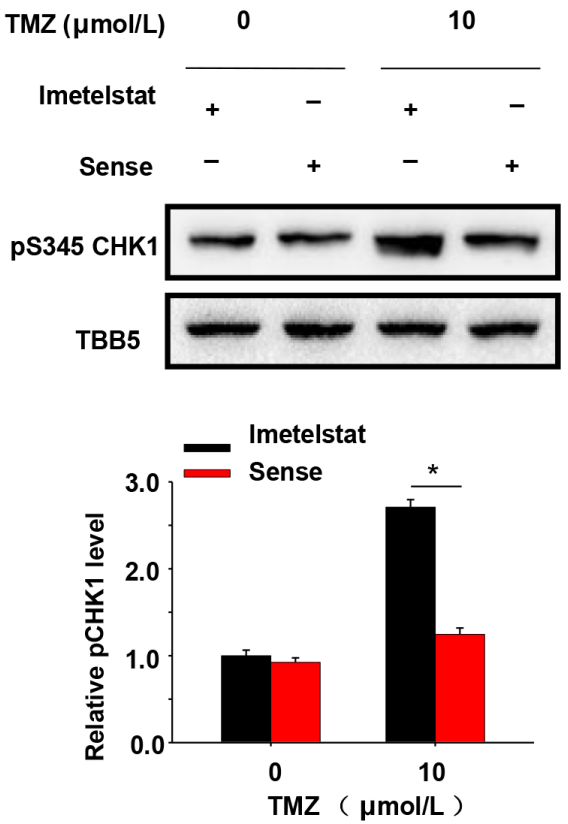
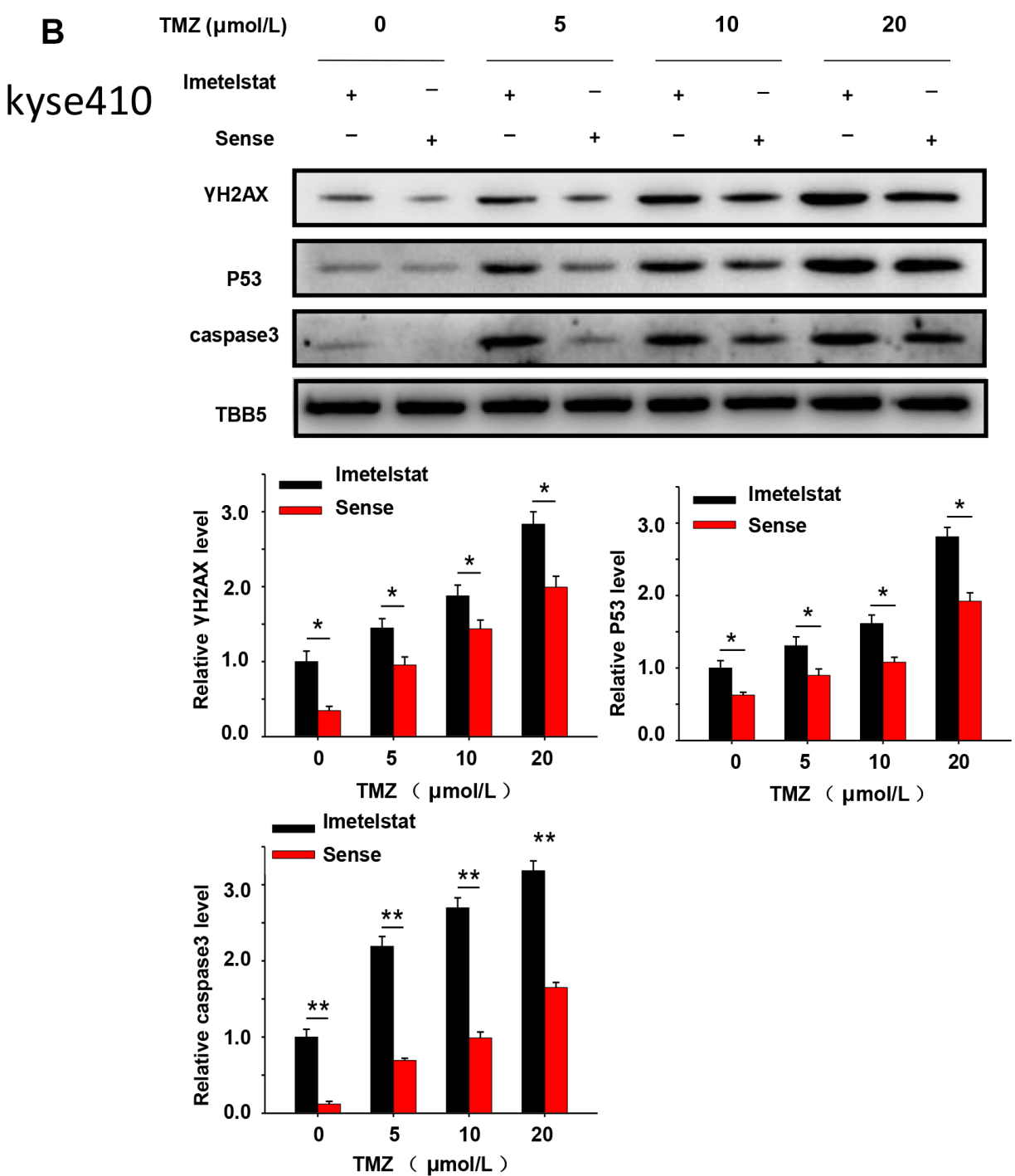

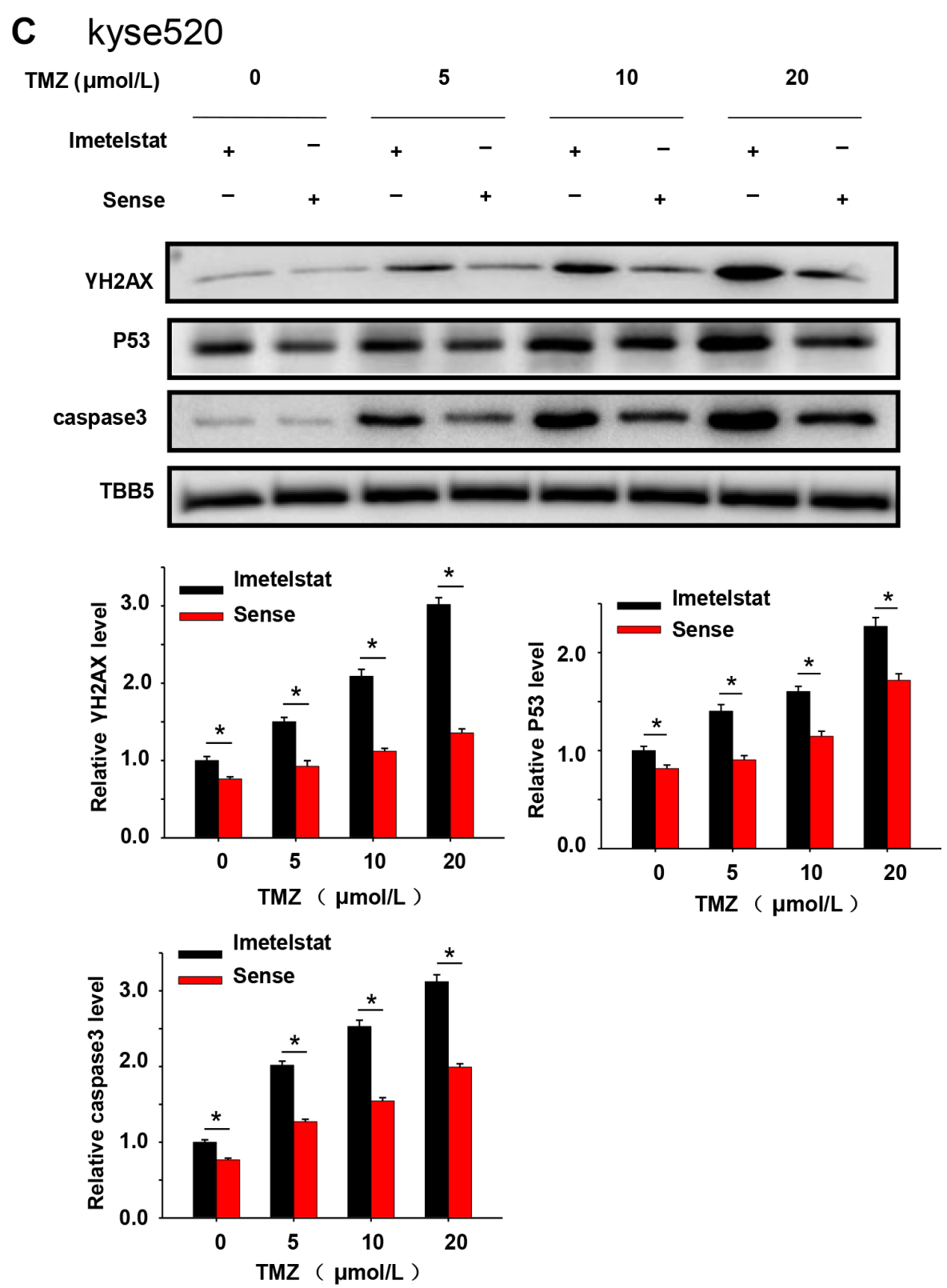

Figure 3: DNA repair and apoptosis signaling protein were upregulated by the treatment of imetelstat in Kyse410 and Kyse520 cells. (A) Kyse410 and Kyse520 cells were treated with $5 \mu \mathrm{M}$ imetelstat or $5 \mu \mathrm{M}$ sense for $72 \mathrm{~h}$ and meanwhile exposed to $10 \mu \mathrm{M}$ TMZ. The expressions of phosphorylation of CHK1 at S345 were detected, taking the expression of TBB5 as inner control. (B and $\mathbf{C})$ Kyse 410 and Kyse 520 cells were treated with $5 \mu \mathrm{M}$ imetelstat or $5 \mu \mathrm{M}$ sense for $72 \mathrm{~h}$ together with $\mathrm{TMZ}(0,5,10,20 \mu \mathrm{M})$. Expression levels of $\gamma$-H2AX, p53 and caspase 3 were detected by immunoblotting. The intensity of each protein band was quantified using the BandScan software (Glyko) and normalized against tublin. *represents statistical significant $(p<0.05)$.

regulation of $\mathrm{Ki} 67$ and imetelstat enables tumor more sensitive to radiation treatment, which was revealed by weaker expression of Ki67 (Figure 5). As observed in Figure 5, the staining of $\mathrm{Ki} 67$ in sense or imetelstat treated group was stronger compared with that exposed to radiation. More importantly, tumor pretreated with sense combined irradiation displayed higher level of Ki67 compared with that in the imetelstat/radiation group, which indicated radiosensitization effect of imetelstat. These findings suggested that imetelstat inhibited tumor growth in mice subjected to radiation was associated with the enhanced apoptosis and suppressed cell proliferation in cancer cells.

\section{DISCUSSION}

Telomerase activation is considered as a key step in cell immortalization and tumorigenesis [15]. It has been reported that positive rate of telomerase activity is $80 \%$ to $90 \%$ in squamous cell carcinoma, which is much higher 
than those in the surrounding normal tissues [16]. This discrepancy between cancer and normal cells provides a considerable therapeutic window for telomerase suppression-based therapy.

Our prior work has verified that imetelstat blocks telomerase activity in esophageal cancer cells, decreases cell proliferation and colony formation ability. More importantly, our research group has reported imetelstat increases radiation induced DNA breaks, which provided theoretical foundation for further researches [9]. In this study, we found that long-term imetelstat treatment could radiosensitize Kyse cells in both vitro and vivo. This is consistent with one study on breast cancer cells, in which long-term treatment of imetelstat showed radiosensitization activity in vitro colony formation assay and in vivo tumor growth assay [17]. Further analysis showed that SER was larger for Kyse410 than Kyse520, 1.9 and 1.6 respectively. These results indicated that Kyse520 is more radioresistant than Kyse410 cells, consisting with our previous results that radiation-induced DSB foci in Kyse410 are more in number and larger in size compared with the DSB foci in Kyse520 cells [9].

Multiple DNA damage repair pathways are implicated in maintaining the genetic integrity of a cell after its exposure to radiation. Wong et al. reported that telomerase deficient mouse developed a radio-sensitivity syndrome because of the delayed DNA break repair [18]. Our previous study reported that treatment of imetelstat and radiation showed synergistic increase and prolonged higher expression of DSB foci compared to cells treated with sense/radiation [9]. In this study, western blot results showed that increased expression of phosphoCHK1 and $\gamma-\mathrm{H} 2 \mathrm{AX}$ in imetelstat treatment group which reflect the magnitude and rate of DSB repair. Our results are consistent with one report in glioblastoma cells that increased levels of $\gamma-\mathrm{H} 2 \mathrm{AX}$ after imetelsat treatment [19]. Cells react to DSBs by triggering the DNA damage signaling, which will further activate intracellular pathways including apoptotic signaling [20-22] One our previous research reported that 17-AAG sensitize esophageal squamous cells to radiation by inhibiting cell proliferation and promote cell apoptosis [23]. Ding et al. reported that sunitinib increased radiation induced DNA double-strand breaks and promoted the apoptosis of human esophageal squamous cell carcinoma cells [24]. Our study showed that imetelstat combined TMZ which mimic the function of radiation could significantly increase cell apoptosis compared with sense/TMZ treatment (Figure 2). Western blot demonstrated that imetelstat treatment could increase expressions of caspase 3 and p53, which promoted cell apoptosis and reflected increased DNA repair signaling (Figure 3B and 3C). Apoptotic cells

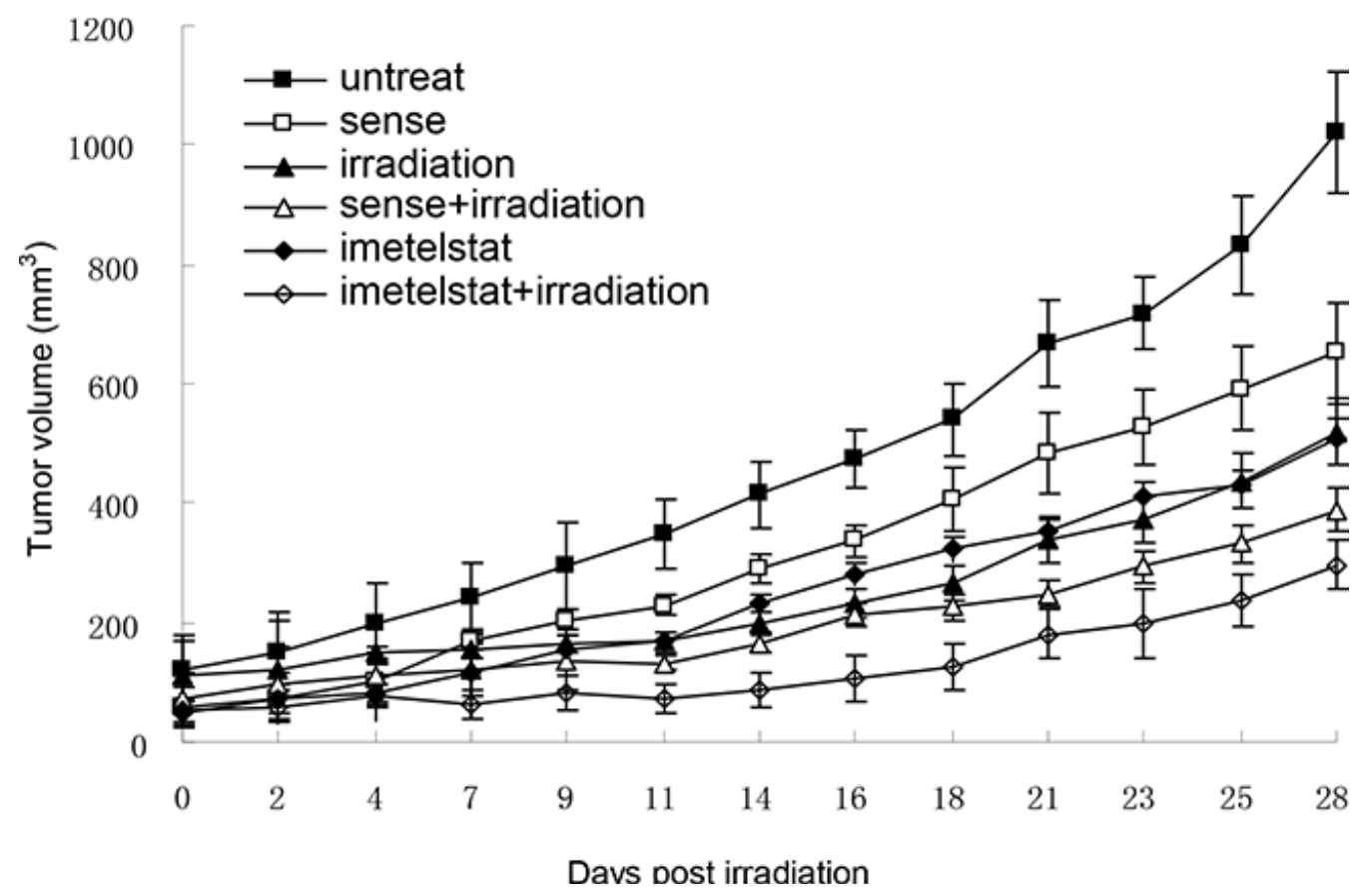

Figure 4: Imetelstat sensitizes esophageal cancer cell Kyse520 to radiation in vivo. Kyse520 cells were pretreated with imetelstat or sense for 7 weeks before injected into nude mice to form tumors. When the size of tumor reached approximately $50 \mathrm{~mm}^{3}$, mice were randomly divided into 6 different groups, including untreated group, radiation group, sense group, imetelstat group, sense + radiation group and imetelstat + radiation group. Tumors in nude mice were subjected to 2 Gy of irradiation for 5 consecutive days to mimic a fractionated weekly scheme. The sizes of tumor were measured 3 times per week to obtain cell growth curve. At days 13,31 and 45 postinjection, imetelstat were injected intraperitoneally to ensure inhibition of telomerase activity. Data are presented as mean tumor volume $\pm \mathrm{SE}$. Imetelstat with irradiation significantly decreased tumor growth compared with mice receiving irradiation alone or irradiation with sense $(p<0.05)$. 
labeled by TUNEL assay in tumor tissue were increased in imetelstat treatment group which is further proved by caspase 3 immunofluorescence staining (Figure 5).

Although in vitro data provide an important preliminary background to advance the potential use of imetelstat, these results must be confirmed and validated in vivo. The clinical application of therapeutic irradiation relies on fractionated does of radiation that are given daily for several weeks. In our study, we extended in vivo results to a fractionated weekly scheme consistent with clinical treatment protocols. Our experiments demonstrated that imetelstat combine with radiation induce tumor growth delay compared to imetelstat treatment or sense/radiation treatment. The results comfirmed that imetelstat can radiosensitize esophageal cancer in vivo. As a marker of cell proliferation, antigen Ki67 was also detected to confirm the tumor growth delay induced by imetelstat treatment.

In conclusion, imetelstat increases radiation sensitivity in esophageal cancer by inducing cell apoptosis and decreasing cell proliferation. In vitro and vivo results support imetelstat as a promising adjuvant cancer treatment in combination with radiation therapy.

\section{MATERIALS AND METHODS}

\section{Main reagents and kits}

The human ESCC (esophageal squamous cell carcinoma) cell Kyse410 and Kyse520 (Deutsche Sammlung von Mikroorganismenund Zellkulturen $\mathrm{GmbH}$, Braunschweig, Germany) were cultivated in PRMI-1640 medium at $37^{\circ} \mathrm{C}$ in a humidified $5 \% \mathrm{CO} 2$ incubator, supplemented with $10 \%$ fetal bovine serum, $100 \mathrm{U}$ penicillin and streptomycin. Primary antibodies against $\gamma$-H2AX, p53, caspase3, and pS345-CHK1 were purchased from Cell Signaling Technology (Beverly, MA). Anti-Ki-67 and tublin antibodies was purchased from Santa Cruz Biotechnology (Santa Cruz, CA). PRMI1640 medium was from GIBCO and fetal bovine serum (FBS) was purchased from Hyclone (Logan, UT, USA).

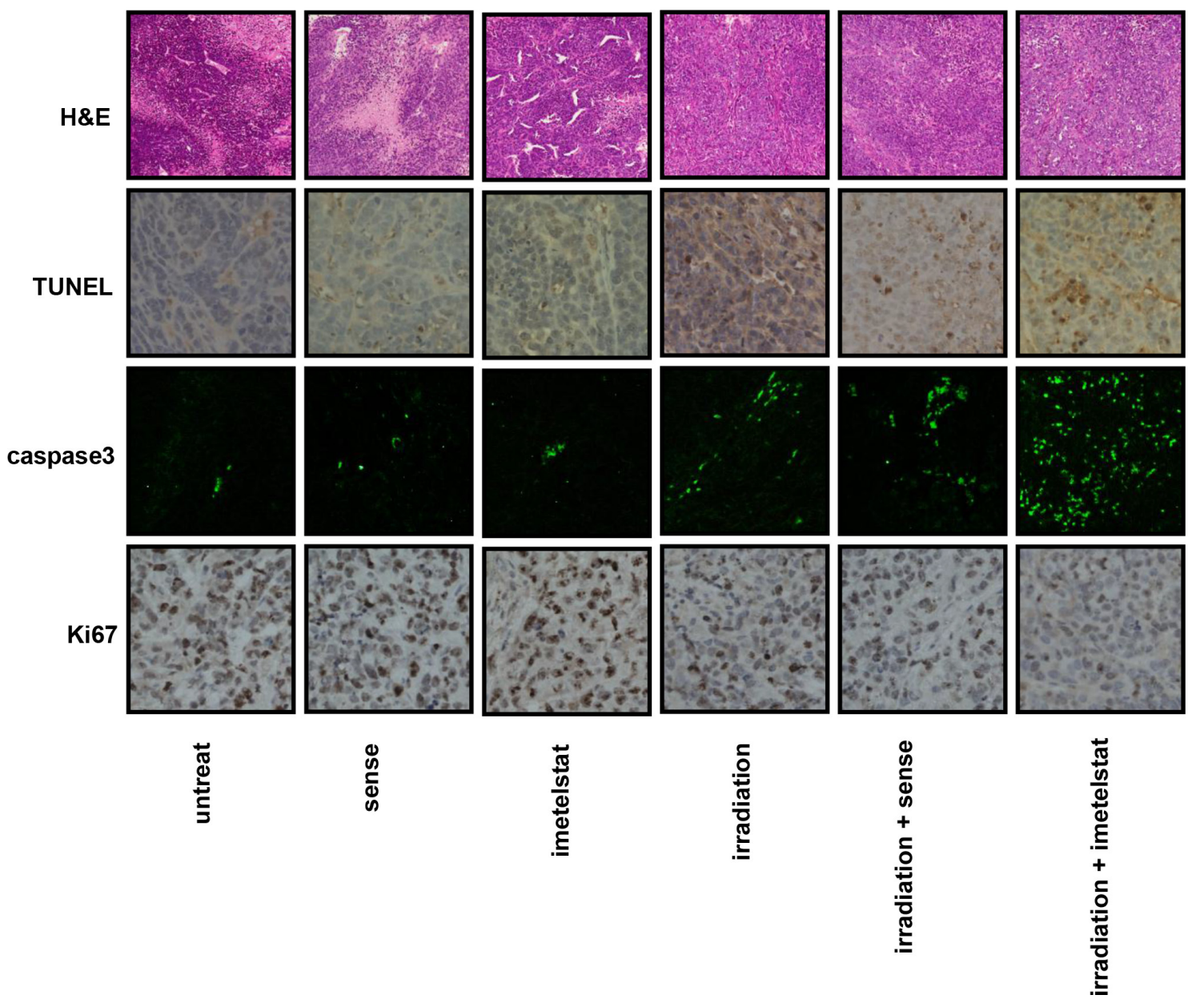

Figure 5: Imetelstat increases cell apoptosis and decreases cell proliferation in vivo. Pathological changes of esophageal squamous carcinoma were evaluated by H\&E staining. Apoptotic cells were labeled by TUNEL assay and further evaluated by immunofluorescence staining of caspase 3. Imetelstat promoted radiation-induced cell apoptosis compared with radiation-treated tumor. The cell proliferation was evaluated by Ki67 staining in tumor tissue. Imetelstat combined with radiation showed less Ki67 expression compared to other treatment groups. 
Penicillin and streptomycin were purchased from Amresco Inc (Solon, OH).

\section{Radiosensitization experiment}

Kyse410 and Kyse520 were pretreated with imetelstat and sense at a concentration of $1 \mu \mathrm{M}$ for 40 days. During this time, cells were passaged every three days and fresh antagonist was added. After that, cells were exposed to radiation of $0,2,4,6$ and $8 \mathrm{~Gy}$ of $6 \mathrm{MeV}$ X-ray at room temperature, respectively. To assay clonogenic cell survival, cells were trypsinized and plated onto $10-\mathrm{cm}$ tissue culture dishes. Cells were incubated for 10-12 days, fixed with ethanol and stained with haematoxylin. Cells able to form a colony of at least 50 cells were considered as clonogenic cells. Survival data were fitted to $S=\exp$ $\left(-\alpha D-\beta D^{2}\right)$ where $D$ is the dose in Gy and $\alpha$ and $\beta$ are fitting parameters. Radiosensitivity was quantified as area under curve (AUC) and the effect of imetelstat on radiosensitivity is presented as sensitizer enhancement ratio (SER), defined as AUC control $/ \mathrm{AUC}_{\text {treated }}$.

\section{Apoptosis assay}

Kyse410 and Kyse520 cells were incubated and treated with $5 \mu \mathrm{M}$ imetelstat or $5 \mu \mathrm{M}$ sense for $72 \mathrm{~h}$ and together with $10 \mu \mathrm{M}$ temozolomide (TMZ). Cells were harvested, washed, resuspended in PBS and then stained with the Annexin V/PI Cell Apoptosis Detection Kit (Franklin Lakes, NJ) according to the manufacturer's instructions. Data acquisition and analysis were conduct with a Becton-Dickinson FACS Calibur flow cytometer using Cell-Quest software (BD Biosciences, Franklin Lakes, NJ).

\section{Western blot}

Kyse410 and Kyse520 cells were incubated and treated with $5 \mu \mathrm{M}$ imetelstat or $5 \mu \mathrm{M}$ sense for $72 \mathrm{~h}$ and meanwhile exposed to increased concentration of TMZ $(0,5,10,20 \mu \mathrm{M})$. Subsequently, proteins in Kyse410 and Kyse520 cells were extracted with RIPA lysis buffer containing a protease inhibitor cocktail, PMSF for $30 \mathrm{~min}$ on ice respectively and then centrifuged at $12000 \mathrm{rpm}$ for $5 \mathrm{~min}$ at $4^{\circ} \mathrm{C}$. Total protein concentration in supernatant of cell lysates was determined by Pierce BCA protein assay kit (Thermo, Massachusetts, USA). Then we used $\gamma$-H2AX, p53, caspase 3 and pS345-CHK1 Abs in the following SDS-PAGE and Western blot detection. Antigens were detected after incubation with an HRPconjugated secondary antibody followed by visualization using an ECL detection system. The intensity of each protein band was quantified using the BandScan software (Glyko) and normalized against tublin in the same samples blotted by anti-tublin antibody.

\section{Animals care and experimental protocol}

All animal maintenance and experimental procedures were carried out in accordance with the National Institutes of Health Guide for the Care and Use of Laboratory Animals. Male nude mice were purchased from Beijing HFK bioscience Company Limited. Mice were maintained in an air-conditioned pathogen-free room under conditions of controlled lighting of $12 \mathrm{~h}$ light/day. Five million Kyse 520 cells cultured were cultured for 7 weeks adding $1 \mu \mathrm{M}$ imetelstat or sense. Esophageal squamous Kyse520 cell suspension was injected subcutaneously into right hind limb groin of nude mice to induce the formation of tumor. When the size of tumor reached approximately $50 \mathrm{~mm}^{3}$, mice were randomly divided into 6 different groups, including untreated group, radiation group, sense group, imetelstat group, sense + radiation group and imetelstat + radiation group. After that, tumors in nude mice were subjected to 2 Gy of irradiation for 5 consecutive days, while other parts were protected by lead. The sizes of tumor were measured 3 times per week to obtain cell growth curve. Mice were sacrificed 50 days later and tumors were dissected subsequently for further analysis. At days 13, 31 and 45 postinjection, inhibitor imetelstat $(30 \mathrm{mg} / \mathrm{kg})$ and control sense $(30 \mathrm{mg} / \mathrm{kg})$ were injected intraperitoneally to ensure inhibition of telomerase activity.

\section{TUNEL assay}

DNA fragments in tissue sections were detected using TUNEL assay kit (BD Pharmingen, CA) according to the manufacturer's instructions. Briefly, the enzyme terminal deoxynucleotidyl transferase (TdT) was used to incorporate digoxigenin-conjugated dUTP at the ends of DNA fragments. The signal of TdT-mediated dUTP nickend labeling was then detected using an anti-digoxigenin antibody conjugated with peroxidase. TUNEL slides were photographed with a confocal laser scanning microscope (Fluoview FV1000, Olympus, Tokyo, Japan). Positive staining of cells within each slide were measured and expressed as a ratio of apoptotic cells to total number of tumor cells.

\section{Immunohistochemistry and immunofluorescence assay}

Tumor tissues were fixed in $10 \%(\mathrm{~V} / \mathrm{V})$ neutral buffered formalin solution for at least $24 \mathrm{~h}$ at room temperature, dehydrated in graded ethanol, cleared in xylene and embedded in paraffin. Perpendicular tumor sections ( $4 \mu \mathrm{m}$ thickness) were mounted on glass slides and de-waxed. Then, tissue sections were prepared for Hematoxylin-Eosin (HE) staining. Pathological changes were observed by light microscopy. The expression of caspase 3 in tumor was detected by immunofluorescence 
assay. Tumor tissues was processed and mounted on glass slides. Briefly, sections were pre-incubated with $1 \%$ Triton $\mathrm{X}-100$ and $10 \%$ bovine serum albumin (BSA) in phosphate buffered saline (PBS) for $1 \mathrm{~h}$ at room temperature. After that, samples were incubated with specific antibodies against caspase 3 for $2 \mathrm{~h}$ at room temperature. Fluorescent secondary antibodies were performed for another $1 \mathrm{~h}$ at room temperature according to the manufacturer's instructions. The expression of Ki67, proliferation cellassociated nuclear antigen in tumor tissues, was assessed by immunohistochemistry. The paraffin sections were heat fixed, deparaffinized in xylene, rehydrated by graded ethanol to distilled water, boiled in sodium citrate buffer and incubated in 3\% hydrogen peroxide after cooling. Each slide was blocked with 3\% BSA at room temperature prior to the incubation with primary antibody overnight at $4^{\circ} \mathrm{C}$, secondary antibody for $20 \mathrm{~min}$ at $37^{\circ} \mathrm{C}$ and streptavidin-HRP $20 \mathrm{~min}$ at $37^{\circ} \mathrm{C}$, respectively. Samples were then stained with $\mathrm{DAB}$ and counterstained with hematoxylin. After dehydrating and drying, the sections were mounted with neutral gum.

\section{Statistical analysis}

All data were presented as means \pm standards deviation (SDs). One-way analysis of variance (ANOVA) followed by Tukey multiple comparison test was performed to evaluate differences between groups. The values were considered statistically significantly different at $p<0.05$.

\section{ACKNOWLEDGMENTS AND FUNDING}

This work was supported by National Nature Science Foundation of China (No. 81301938, 81670747), Medical Science and technology development Foundation, Nanjing Department of Health (JQX14007). The work is also supported by Ministry of Science and Technology of China (2013CB911600), Jiangsu Provincial Natural Science Foundation (BK20130044, BK20130061), CJ20160051, The Research Fund for the Doctoral Program of Higher Education of China (RFDP) (20133207110005), The Program for New Century Excellent Talents in University of Ministry of Education of China (NCET13- 0868), the Priority Academic Program Development Award for Jiangsu Higher Education Institutions. We thank Sergei Gryaznov for providing imetelstat and sense.

\section{CONFLICTS OF INTEREST}

The authors declare no conflicts of interest.

\section{REFERENCES}

1. Tirumani H, Rosenthal MH, Tirumani SH, Shinagare AB, Krajewski KM, Ramaiya NH. Esophageal Carcinoma:
Current Concepts in the Role of Imaging in Staging and Management. Can Assoc Radiol J. 2015; 66:130-9. doi: 10.1016/j.carj.2014.08.006.

2. Reeh M, Nentwich MF, Asani S, Uzunoglu FG, Bockhorn M, Sauter G, Rösch T, Izbicki JR, Bogoevski D. Locally advanced esophageal carcinoma: is there still a role of surgery alone without neoadjuvant treatment? J Gastrointest Surg. 2015; 19:587-93. doi: 10.1007/s11605015-2762-y.

3. Sjoquist KM, Burmeister BH, Smithers BM, Zalcberg JR, Simes RJ, Barbour A, Gebski V. Survival after neoadjuvant chemotherapy or chemoradiotherapy for resectable oesophageal carcinoma: an updated meta-analysis. Lancet Oncol. 2011; 12:681-92. doi: 10.1016/S14702045(11)70142-5.

4. Song YP, Ma JB, Hu LK, Zhou W, Chen EC, Zhang W. Phase I/II study of hypofractioned radiation with threedimensional conformal radiotherapy for clinical T3-4N01M0 stage esophageal carcinoma. Technol Cancer Res Treat. 2011; 10:25-30. doi: 10.7785/tcrt.2012.500176.

5. Chang L, Graham P, Hao J, Bucci J, Malouf D, Gillatt D, Li Y. Proteomics discovery of radioresistant cancer biomarkers for radiotherapy. Cancer Lett. 2015; 369:289-97. doi: 10.1016/j.canlet.2015.09.013.

6. Ghorab MM, Ragab FA, Heiba HI, El-Hazek RM. Anticancer and radio-sensitizing evaluation of some new thiazolopyrane and thiazolopyranopyrimidine derivatives bearing a sulfonamide moiety. Eur J Med Chem. 2011; 46:5120-6. doi: 10.1016/j.ejmech.2011.08.026.

7. Harley CB. Telomerase and cancer therapeutics. Nat Rev Cancer. 2008; 8:167-79. doi: 10.1038/nrc2275.

8. Baerlocher GM, Oppliger Leibundgut E, Ottmann OG, Spitzer G, Odenike O, McDevitt MA, Röth A, Daskalakis M, Burington B, Stuart M, Snyder DS. Telomerase Inhibitor Imetelstat in Patients with Essential Thrombocythemia. N Engl J Med. 2015; 373:920-8. doi: 10.1056/NEJMoa1503479.

9. Wu X, Smavadati S, Nordfjäll K, Karlsson K, Qvarnström F, Simonsson M, Bergqvist M, Gryaznov S, Ekman S, Paulsson-Karlsson Y. Telomerase antagonist imetelstat inhibits esophageal cancer cell growth and increases radiation-induced DNA breaks. Biochim Biophys Acta. 2012; 1823:2130-5. doi: 10.1016/j.bbamcr.2012.08.003.

10. Herbert BS, Gellert GC, Hochreiter A, Pongracz K, Wright WE, Zielinska D, Chin AC, Harley CB, Shay JW, Gryaznov SM. Lipid modification of GRN163, an N3' $\rightarrow$ P5' thio-phosphoramidate oligonucleotide, enhances the potency of telomerase inhibition. Oncogene. 2005; 24: 5262-8. doi: 10.1038/sj.onc. 1208760

11. Dikmen ZG, Ozgurtas T, Gryaznov SM, Herbert BS. Targeting critical steps of cancer metastasis and recurrence using telomerase template antagonists, Biochim Biophys Acta-Mol Basis Dis. 2009; 240-7. doi: 10.1016/j. bbadis.2009.01.018. 
12. Shammas MA, Qazi A, Batchu RB, Bertheau RC, Wong JY, Rao MY, Prasad M, Chanda D, Ponnazhagan S, Anderson KC, Steffes CP, Munshi NC, De Vivo I et al. Telomere maintenance in laser capture microdissectionpurified Barrett's adenocarcinoma cells and effect of telomerase inhibition in vivo. Clin Cancer Res. 2008; 14: 4971-80. doi: 10.1158/1078-0432.CCR-08-0473.

13. Raymond AA, Benhamouche S, Neaud V, Di Martino J, Javary J, Rosenbaum J. Reptin regulates DNA double strand breaks repair in human hepatocellular carcinoma. PLoS One. 2015; 10:e123333. doi: 10.1371/journal. pone. 0123333 .

14. Wang B, Matsuoka S, Carpenter PB, Elledge SJ. 53BP1, a mediator of the DNA damage checkpoint. Science. 2002; 298:1435-8. doi: 10.1126/science.1076182.

15. Takubo K, Nakamura K, Izumiyama $N$, Mafune $K$, Tanaka Y, Miyashita M, Sasajima K, Kato M, Oshimura M. Telomerase activity in esophageal carcinoma. J Surg Oncol. 1997; 66:88-92. doi:10.1002/(SICI)10969098(199710)66:2<88: AID-JSO3>3.0.CO;2-H.

16. Shay JW, Bacchetti S. A survey of telomerase activity in human cancer, Eur J Cancer. 1997; 33:787-91. doi: 10.1016/S0959-8049(97)00062-2.

17. Gomez-Millan J, Goldblatt M, Gryaznov M, Mendonca S, Herbert S. Specific telomere dysfunction induced by GRN163L increases radiation sensitivity in breast cancer cells. Int J Radiat Oncol Biol Phys. 2007; 67:897-905. doi: 10.1016/j.ijrobp.2006.09.038

18. Wong KK, Chang S, Weiler SR, Ganesan S, Chaudhuri J, Zhu C, Artandi SE, Rudolph KL, Gottlieb GJ, Chin L, Alt FW, DePinho RA. Telomere dysfunction impairs DNA repair and enhances sensitivity to ionizing radiation. Nat Genet. 2000; 26:85-88. doi: 10.1038/79232.
19. Marian CO, Cho SK, McEllin BM, Maher EA, Hatanpaa KJ, Madden CJ, Mickey BE, Wright WE, Shay JW, Bachoo RM. The telomerase antagonist, imetelstat, efficiently targets glioblastoma tumor-initiating cells leading to decreased proliferation and tumor growth. Clin Cancer Res. 2010; 16:154-63. doi: 10.1158/1078-0432.CCR-09-2850.

20. d'Adda di Fagagna F, Reaper PM, Clay-Farrace L, Fiegler H, Carr P, Von Zglinicki T, Saretzki G, Carter NP, Jackson SP. A DNA damage checkpoint response in telomere-initiated senescence. Nature. 2003; 426:194-8. doi: 10.1038/nature02118.

21. Masutomi K1, Possemato R, Wong JM, Currier JL, Tothova Z, Manola JB, Ganesan S, Lansdorp PM, Collins K, Hahn WC. The telomerase reverse transcriptase regulates chromatin state and DNA damage responses. Proc Natl Acad Sci. 2005; 102:8222-7. doi: 10.1073/pnas.0503095102.

22. Valerie K, Yacoub A, Hagan MP, Curiel DT, Fisher PB, Grant S, Dent P. Radiation induced cell signaling: insideout and outside-in. Mol Cancer Ther. 2007; 6:789-801. doi: 10.1158/1535-7163.MCT-06-0596.

23. Wu X, Wanders A, Wardega P, Tinge B, Gedda L, Bergstrom S, Sooman L, Gullbo J, Bergqvist M, Hesselius P, Lennartsson J, Ekman S. Hsp90 is expressed and represents a therapeutic target in human oesophageal cancer using the inhibitor 17-allylamino-17-demethoxygeldanamycin. Br J Cancer. 2009; 100:334-43. doi: 10.1038/sj.bjc.6604855.

24. Ding $Y Q$, Zhu HC, Chen XC, Sun XC, Yang X, Qin Q, Zhang H, Yang Y, Yang YH, Gao L, Luo JD, Zhou XF. Sunitinib modulates the radiosensitivity of esophageal squamous cell carcinoma cells in vitro. Dis Esophagus. 2015; 6. doi: 10.1111/dote.12440. [Epub ahead of print] 